\title{
Oxidized Carbon Nanosphere-Based Subunit Vaccine Delivery System Elicited Robust Th1 and Cytotoxic T Cell Responses
}

\author{
Pritsana Sawutdeechaikul ${ }^{1,2}$, Felipe $\mathrm{Cia}^{3}$, Gregory J. Bancroft ${ }^{3}$, Supason Wanichwecharungruang ${ }^{4}$, \\ Chutamath Sittplangkoo ${ }^{2,5}$, and Tanapat Palaga ${ }^{1,2,3,4 *}$ \\ ${ }^{1}$ Graduate Program in Microbiology and Microbial Technology, Department of Microbiology, Faculty of Science, Chulalongkorn University, \\ Bangkok 10330, Thailand \\ ${ }^{2}$ Center of Excellence in Immune-mediated Diseases, Chulalongkorn University, Bangkok 10330, Thailand \\ ${ }^{3}$ Department of Immunology and Infection, London School of Hygiene and Tropical Medicine, Keppel Street, London, WC1E 7HT, UK \\ ${ }^{4}$ Center of Excellence in Materials and Bio-Interfaces, Chulalongkorn University and Department of Chemistry, Faculty of Science, \\ Chulalongkorn University, Bangkok 10330, Thailand \\ ${ }^{5}$ Graduate Program in Biotechnology, Faculty of Science, Chulalongkorn University, Bangkok, Thailand 10330
}

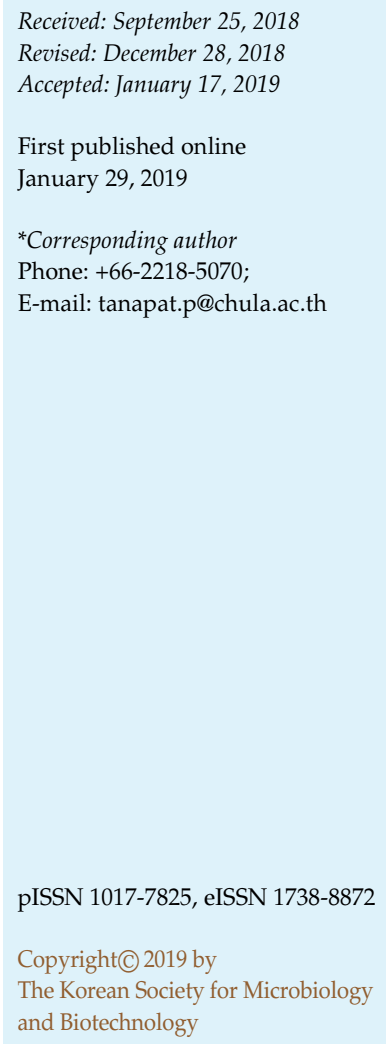

Subunit vaccines are safer and more stable than live vaccines although they have the disadvantage of eliciting poor immune response. To develop a subunit vaccine, an effective delivery system targeting the key elements of the protective immune response is a prerequisite. In this study, oxidized carbon nanospheres (OCNs) were used as a subunit vaccine delivery system and tuberculosis $(\mathrm{TB})$ was chosen as a model disease. TB is among the deadliest infectious diseases worldwide and an effective vaccine is urgently needed. The ability of OCNs to deliver recombinant Mycobacterium tuberculosis (Mtb) proteins, Ag85B and HspX, into bone marrow derived macrophages (BMDMs) and dendritic cells (BMDCs) was investigated. For immunization, OCNs were mixed with the two TB antigens as well as the adjuvant monophosphoryl lipid A (MPL). The protective efficacy was analyzed in vaccinated mice by aerosol $\mathrm{Mtb}$ challenge with a virulent strain of Mtb and the bacterial burdens were measured. The results showed that OCNs are highly effective in delivering Mtb proteins into the cytosol of BMDMs and BMDCs. Upon immunization, this vaccine formula induced robust Th1 immune response characterized by cytokine profiles from restimulated splenocytes and specific antibody titer. More importantly, enhanced cytotoxic $\mathrm{CD}^{+} \mathrm{T}$ cell activation was observed. However, it did not reduce the bacteria burden in the lung and spleen from the aerosol Mtb challenge. Taken together, OCNs are highly effective in delivering subunit protein vaccine and induce robust Th1 and $\mathrm{CD}^{+} \mathrm{T}$ cell response. This vaccine delivery system is suitable for application in settings where cell-mediated immune response is needed.

Keywords: Carbon nanospheres, vaccine delivery, subunit vaccine, cytotoxic T lymphocyte; tuberculosis

\section{Introduction}

Mycobacterium tuberculosis (Mtb) is an intracellular bacterial pathogen that is the causative agent of tuberculosis (TB). Currently, TB is still one of the major global health problems and one of the leading causes of death among infectious diseases [1]. The live attenuated strain of
Mycobacterium bovis, Bacillus Calmette-Guérin (BCG), has been the only TB vaccine available for human use since 1921. It provides protection against severe forms of TB in children but confers less protective efficacy against pulmonary TB in adults [2]. Therefore, an effective vaccination strategy and novel TB vaccines are urgently needed to stop TB. 
Cell-mediated immune response (CMI), such as type I helper T cells (Th1) and cytotoxic T lymphocytes (CTL) are believed to play a major role in defending against $\mathrm{Mtb}$ [3, 4]. Therefore, this type of immune response became the main focus for developing new TB vaccines. Antigenpresenting cells (APC) process and present antigens to $\mathrm{T}$ cells and the outcome of the responses are determined by various factors including route of antigen uptake and cytokine milieu. Conventionally, endogenous antigens (cytosolic antigen) are processed and loaded onto major histocompatibility complex (MHC) class I molecules and then presented to $\mathrm{CD}^{+} \mathrm{T}$ cells [5]. However, crosspresentation, which is an alternative pathway, also allows the loading of exogenous antigens on to MHCI [5, 6]. This mechanism is important for designing subunit vaccines to elicit CMI and $\mathrm{CD}^{+} \mathrm{T}$ cell responses. Subunit vaccines have increasingly become an important strategy due to a high degree of safety and their standardized production [1]. Therefore, proper adjuvants and novel delivery systems are increasingly needed with the aim to increase immunogenicity of peptides or recombinant antigens.

Recently, nanoparticle-based vaccine delivery systems have become widely studied [7-9]. Nanoparticles have many advantages for vaccine development. First, the they size of nanoparticles is in the range of microorganisms and can be targeted and readily ingested by APCs. As a result, nanoparticles help to increase antigen presentation to immune cells [10]. Second, encapsulated antigens can be protected from rapid degradation by various enzymes in the environment before being taken up by APCs and antigen release can be controlled to prolong presentation by APCs [11-13]. Third, as soluble antigens are less suitable to generate cross-presentation, combining of nanoparticles with soluble antigen can generate a particulate form that shows higher levels of cross-presentation than the soluble antigens [14,15]. Therefore, using nanoparticles as a vaccine delivery system is highly attractive and promising for TB vaccine development.

To date, nanoparticles including viral vector and liposome have been applied in clinical trials of TB subunit vaccines. For viral vectored vaccines, recombinant adenovirus, vaccinia virus and influenza virus are used for expressing Mtb antigen. In preclinical studies, polymeric nanoparticles such as poly (lactide-co glycolide) (PLGA), chitosan, poloxamer and pluronic-stabilized polypropylene sulfide are also reported [16].

Oxidized carbon nanospheres (OCNs) are water dispersible, stable, negatively charged particles with a diameter of around $130 \mathrm{~nm}$. OCNs can be prepared through a special oxidation-exfoliation of inexpensive graphite or graphene nanoplates [17]. This low toxic and biocompatible nanoparticle possesses excellent ability to deliver macromolecules into cells $[17,18]$. It has been proposed that OCNs can generate transient pores on lipid bilayer membranes, thus allowing nearby entities to leak into cells. In some cells that can endocytose OCNs effectively, the endocytosed OCNs could also leak the endosomes $[17,18]$. As a result, OCNs seem to be a perfect means of delivery to bring big antigen into the cytosol of cells.

Once the antigens are in the cytosol, the chance of antigen being loaded onto MHC I is expected to improve, leading to enhanced $\mathrm{CD} 8^{+} \mathrm{T}$ cell activation, but to date, this type of vaccine delivery system has not been described for Mtb antigens. Overall, the application of OCNs as a delivery system for TB subunit vaccine is promising. To the best of our knowledge, carbon-based nanoparticles for TB vaccine development have never been reported before.

In this study, we tested the hypothesis that an OCNbased delivery system induces better Th1/CTL response using Mtb antigens as a model. We prepared our prototype TB vaccine by mixing three components of i) OCN, ii) two dominant Mtb antigens, Ag85B (a fibronectin-binding protein with mycolyltransferase activity, Rv1886c) and HspX (a heat shock protein, Rv2031c) and iii) monophosphoryl lipid A (MPL), a potent Th1 adjuvant [19]. Ag85B and HspX represent replicating state and dormant state proteins [20, 21], respectively. The ability of OCNs to deliver $\mathrm{Mtb}$ antigens in to macrophages and dendritic cells (DC) was tested in vitro. Immunogenicity of the recombinant vaccine was evaluated by antibody titer and in vitro splenocyte restimulation assay. Finally, the protective efficacy was tested by aerosol challenge with Mtb.

\section{Materials and Methods}

\section{Reagents}

Dulbecco's Modified Eagle Medium (DMEM), sodium pyruvate, HEPES and penicillin-streptomycin were purchased from GE Healthcare Life Sciences (Logan, USA). Roswell Park Memorial Institute (RPMI) 1640 Medium, Glutamax and Fetal bovine serum (FBS) were purchased from invitrogen (USA). Horse serum was purchased from Thermo Fisher Scientific (UK). MPL was vaccine grade and purchased from (Invivogen, USA). Recombinant proteins Ag85B and HspX were obtained from BEI Resources (Manassas, USA).

\section{Animals}

BALB/c or C57/BL6 female mice (8 weeks old) were purchased from Nomura Siam International (Thailand). All experiments 
involving mice were approved by the Chulalongkorn University Institutional Animal Care and Use Committee (CU-IACUC) (No.1673005). For Mtb challenge, CB6F1/Crl female mice (6-8 weeks old) were used. All the experiments were performed following the ethical and legal requirements set by the UK Home Office guidelines referring to the welfare of experimental animals.

\section{Preparation of BMDMs and BMDCs}

Bone marrow cells were isolated from tibias and femurs of $\mathrm{BALB} / \mathrm{c}$ female mice by flushing and BMDMs were generated by incubating cells in BMDM media (DMEM supplemented with 10\% (v/v) FBS, 1\% (w/v) sodium pyruvate, $1 \%(\mathrm{w} / \mathrm{v})$ HEPES, $100 \mathrm{U} / \mathrm{ml}$ penicillin, $100 \mathrm{mg} / \mathrm{ml}$ streptomycin, 20\% L929 cell-conditioned media and $5 \%$ horse serum), followed by culture in this media for a week. Media were changed every 3 days [22]. To generate BMDCs, bone marrow cells were cultured in RPMI supplemented with $10 \%$ (v/v) FBS, $100 \mathrm{U} / \mathrm{ml}$ penicillin, $100 \mu \mathrm{g} / \mathrm{ml}$ streptomycin, $20 \mathrm{mM}$ Glutamax, $10 \mathrm{ng} / \mathrm{ml}$ granulocyte-macrophage colonystimulating factor (GM-CSF) and $10 \mathrm{ng} / \mathrm{ml} \mathrm{IL}-4$ for a week. Half of the culture media was replaced every 2 days [23].

\section{Formulation of OCNs with Recombinant Antigens}

OCN was prepared as described previously [17]. OCN was autoclaved at $121^{\circ} \mathrm{C}$ and sonicated for $5 \mathrm{~min}$ prior to use. $\mathrm{OCN}+\mathrm{Ag} 85 \mathrm{~B}+\mathrm{HspX}$ mixture was prepared by mixing OCNAg85B complex with OCN-HspX complex. Briefly, two $\mu g$ of OCN was mixed with $2 \mu \mathrm{g}$ of each protein separately in water and the total volume of each complex was $5 \mu$. The two mixtures were incubated overnight at $4^{\circ} \mathrm{C}$ before being formulated into a twoprotein mixture together. Five microliters of mixture were added in $500 \mu \mathrm{l}$ DMEM completed media (DMEM supplemented with $10 \%(\mathrm{v} / \mathrm{v})$ FBS, $1 \%(\mathrm{w} / \mathrm{v})$ sodium pyruvate, $1 \%(\mathrm{w} / \mathrm{v})$ HEPES, $100 \mathrm{U} / \mathrm{ml}$ penicillin, $0.25 \mathrm{mg} / \mathrm{ml}$ streptomycin) to obtain the final weight of $2 \mu \mathrm{g}$ of OCN, $1 \mu \mathrm{g}$ of Ag85B and $1 \mu \mathrm{g}$ of HspX. The weight ratio of $\mathrm{OCN}$ to proteins was 1:1.

\section{Immunofluorescent Staining}

BMDMs and BMDCs were seeded at $1 \times 10^{5}$ and $2 \times 10^{5}$ cells / well on 8-well chamber slides (Thermo Fisher Scientific, USA), respectively. BMDM cells were grown overnight in BMDM media, while BMDCs were cultured in RPMI media supplemented with GM-CSF, IL-4 and other supplements as described above. Cells were incubated at $37^{\circ} \mathrm{C}$ with $5 \% \mathrm{CO}_{2}$ for overnight. For BMDMs, media were changed to complete DMEM and incubated further overnight, and the culture supernatants were replaced with OCN formulation in complete DMEM. For BMDCs, half of the culture media was replaced by RPMI media without cytokines, and further incubated for $2 \mathrm{~h}$, before the OCN formulation was added. Cells were maintained at $37^{\circ} \mathrm{C}, 5 \% \mathrm{CO}_{2}$ for $1 \mathrm{~h}$ and $2 \mathrm{~h}$. Cells were fixed, permeabilized and stained with standard immunofluorescent staining protocol. Ag85B staining was performed by using 1:200 dilution of rabbit polyclonal anti-Ag85B (a kind gift from Prof. Watchara Kasinrerk, Faculty of Associated Medical Sciences,
Chiang Mai University, Thailand) and detected with 1:500 dilution of Alexa 555-labeled anti-rabbit IgG (Cell Signaling Technology, USA), while HspX was stained with 1:200 dilution of monoclonal anti-HspX (BEI Resources, USA) and detected with 1:500 dilution of Alexa 488-labeled anti-mouse IgG (Cell Signaling Technology) for $1 \mathrm{~h}$ each. Nuclei were stained with (Invitrogen). Confocal microscopy was used for observing the intracellular uptake (Olympus FV10i, Japan).

\section{Vaccine Preparation (HspX+Ag85B+OCN+MPL)}

One hundred microliters of vaccine per mouse were prepared by mixing OCN-Ag85B with OCN-HspX and MPL (Invivogen) together. Briefly, each mixture was prepared by mixing $10 \mu \mathrm{g}$ of OCN with $10 \mu \mathrm{g}$ of Ag85B or $10 \mu \mathrm{g}$ of HspX separately (OCN: protein weight ratio was 1:1) in endotoxin-free water at $4^{\circ} \mathrm{C}$ for overnight in $45 \mu \mathrm{l}$. After that, the two mixtures were mixed together and MPL $(10 \mu \mathrm{g})$ was added to reach the final volume of $100 \mu \mathrm{l}$. The final amounts of OCN, Ag85B and HspX in $100 \mu \mathrm{l}$ of vaccine were 20,10 , and $10 \mu \mathrm{g}$, respectively.

\section{Immunization Strategy and Challenge of Mice with Mtb}

To study the immunogenicity of the prototype TB vaccine, 8week-old BALB/c female mice ( $n=6$ per group) were used. The day before the immunization, blood was collected from the facial vein. One hundred microliters of formula including saline, $\mathrm{HspX}+\mathrm{Ag} 85 \mathrm{~B}+\mathrm{MPL}$ and $\mathrm{Hsp} \mathrm{X}+\mathrm{Ag} 85 \mathrm{~B}+\mathrm{OCN}+\mathrm{MPL}$ were administered 3 times subcutaneously at 2 -week intervals. The animals were sacrificed at day 35 to collect blood and spleens.

For protective efficacy, two immunization strategies were performed to test the protective efficacy of vaccine. First, mice were primed and boosted with the prototype vaccine. Second, mice were primed with BCG and boosted with prototype vaccine later. Six-to-eight-week-old CB6F1/Crl female mice $(n=6$ per group) were used. Vaccinations were performed subcutaneously using a dose volume of $100 \mu \mathrm{l}$. BCG $1331\left(5 \times 10^{6} \mathrm{CFU} /\right.$ mouse $)$ was used as the "gold standard" control for vaccinations and to prime the mice. In both testing approaches, mice were subjected to an aerosol challenge with approx. 100 CFU/mouse of Mycobacterium tuberculosis H37rv, six weeks after last vaccination. The body weight of the mice was recorded once a week. Lungs and spleens were harvested 6 weeks after challenge, and organ homogenates were prepared and then plated for CFU counting.

\section{Splenocyte Restimulation Assay}

Splenocytes were collected by homogenization of spleens through $100-\mu \mathrm{m}$ cell strainers with the syringe plunger. Red blood cell lysis buffer was used to lyse red blood cells. Splenocytes were seeded at $4 \times 10^{6}$ cells/well, total volume of $1 \mathrm{ml}$ in a 24-well plate. Specific antigen including Ag85B and $\mathrm{HspX}$ at the final concentration of $10 \mu \mathrm{g} / \mathrm{ml}$ were added. For measurement of cytokine profiles by ELISA, incubation time for IL-2 detection was $48 \mathrm{~h}$ while IL-5 and IFN- $\gamma$ detection were $72 \mathrm{~h}$. Splenocytes from each mouse were restimulated separately by each antigen and the culture 
supernantant was subjected to ELISA in duplicate. In the case of intracellular granzyme B staining, incubation time was $72 \mathrm{~h}$ and in the last $4 \mathrm{~h}, 1 \mu \mathrm{l}$ of Golgi plug (Brefeldin A) (BD Biosciences, USA), was added to the cultures.

\section{Specific Antibody Titer}

Serum was collected to measure the level of specific total IgG, IgG1 and IgG2a. Two ug/ml (100 $\mu$ l per well) of Ag85B or HspX were coated on a 96-well MaxiSorp plate (Nunc, Denmark) and incubated at $4{ }^{\circ} \mathrm{C}$ overnight. Plates were washed and blocked with $10 \%$ FBS in PBS and serial dilutions of serum were added. After $1 \mathrm{~h}$ of incubation, plates were washed and sheep anti-mouse IgGHRP (GE Healthcare, UK) or rabbit anti-mouse IgG1-HRP (Invitrogen, USA) or rat anti-mouse IgG2a-HRP (Invitrogen) were added. After washing, 3,3 ,5,5'-tetramethylbenzidine (Sigma Aldrich, USA) was added to develop the signal and $1 \mathrm{~N} \mathrm{H}_{2} \mathrm{SO}_{4}$ was used to stop the reaction. The optical density (OD) at $450 \mathrm{~nm}$ was measured with microplate reader (Anthos 2010, Biochrom, UK).

\section{Intracellular Cytokine Staining}

Cells were processed by using a BD Cytofix/Cytoperm Plus Fixation/Permeabilization Kit (BD Biosciences), according to manufacturer's instructions. Briefly, cells were stained for cell surface markers, CD3 and CD8 with biotin-labeled anti-mouse CD3 $\varepsilon$ (Biolegend, USA) and PE-labeled anti-mouse CD8 $\alpha$ (Biolegend), respectively. PE/Cy7-labeled streptavidin (Biolegend) was used as secondary reagent for detection. After surface staining, cells were fixed, permeabilized and stained for granzyme B using FITC-labeled, anti-human/mouse granzyme B (Biolegend). Isotype matched antibodies were used as a control. Processed cells were first gated on forward and side scatters for viable cell population, followed by gating for $\mathrm{CD} 3$ and CD8. Among the CD3+CD8+ subset, cells were gated for granzyme B. A total of 100,000 events were acquired per sample. Data were collected using a flow cytometer (Beckman Coulter, USA) and analyzed by FlowJo software (TreeStar, USA).

\section{Measurement of Cytokine Production by ELISA}

Cell culture supernatants were collected to measure concentrations of IFN- $\gamma$, IL-2 and IL-5 using LEGEND MAX mouse IFN- $\gamma$, IL-2 and IL-5 ELISA kits (Biolegend) following the manufacturer's protocol. The absorbance was detected at OD $450 \mathrm{~nm}$ using a microplate reader.

\section{Statistical Analysis}

All data were analyzed for statistical significance using GraphPad Prism (version 5.03) software (GraphPad, USA). All data were analyzed by one-way analysis of variance (ANOVA) for determining statistically significant differences between groups. Probability $(p)$ values less than or equal to $0.05,0.01$, and 0.001 were considered as significant and labeled with one, two and three asterisks, respectively.

\section{Results}

\section{Enhancing Mtb Protein Uptake in BMDMs and BMDCs with OCNs}

To test the ability of OCNs to deliver proteins into antigen-presenting cells, recombinant $\mathrm{Mtb}$ proteins mixed with OCN at a weight ratio of 1:1 was tested in BMDMs and BMDCs. Immunofluorescence staining showed that two Mtb proteins, Ag85B and HspX, were observed inside both BMDMs and BMDCs when combined with OCNs at

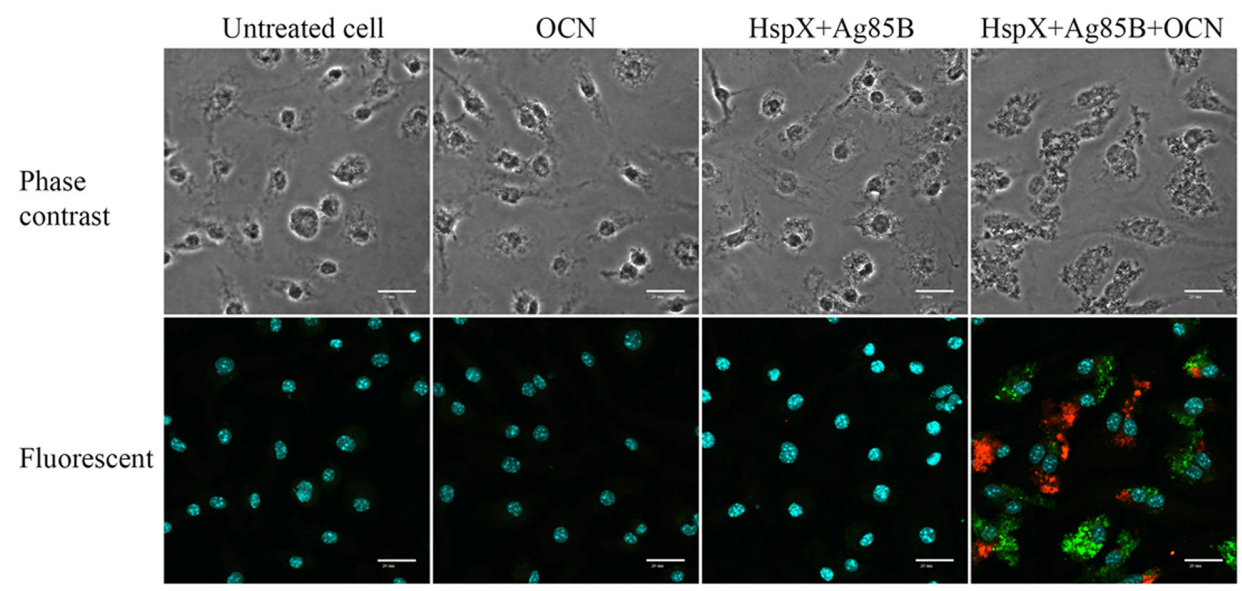

Fig. 1. OCNs increase Mtb antigen protein uptake by macrophages.

Complex of OCN:HspX/Ag85B (weight ratio 1:1) were tested in $1 \times 10^{5}$ of BMDM cells. After $1 \mathrm{~h}$ of incubation at $37^{\circ} \mathrm{C}$, cells were fixed and subjected to an immunofluorescent staining. Ag85B and HspX were stained with rabbit polyclonal anti-Ag85B and monoclonal anti-HspX as a primary antibody, respectively. For detection, anti-rabbit IgG-Alexa 555 (Red) and anti-mouse IgG-Alexa 488 (Green) were used as a secondary antibody. Nuclei were stained with Hoechst (blue). Cells were observed by confocal microscope with 90X magnification. Scale bar $=20 \mu \mathrm{m}$. 


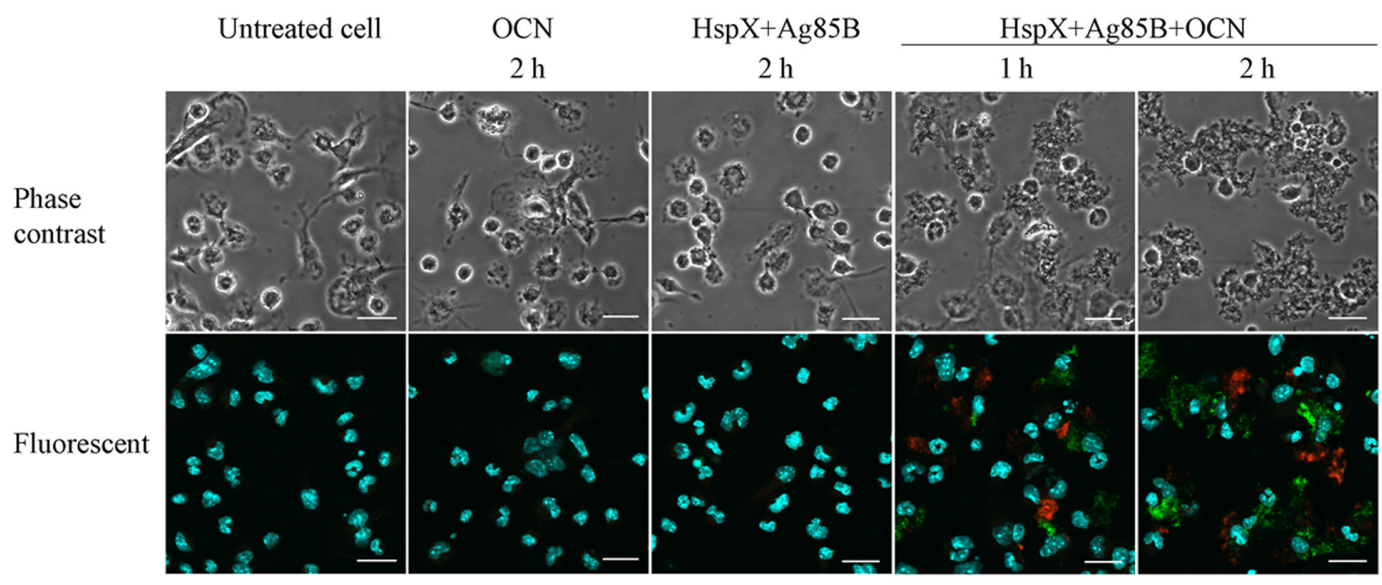

Fig. 2. OCNs have the ability to increase Mtb antigen protein uptake by BMDCs.

The OCN:HspX/Ag85B (weight ratio 1:1) were tested in $2 \times 10^{5}$ of BMDC cells. After 1 or $2 \mathrm{~h}$ of incubation at $37^{\circ} \mathrm{C}$, cells were fixed and subjected to an immunofluorescent staining. Ag85B (red) and HspX (green) were detected as in Figure 1. Nuclei were stained with Hochest (blue). Cells were observed by confocal microscope with $60 \mathrm{X}$ magnification. Scale bar $=20 \mu \mathrm{m}$

$1 \mathrm{~h}$ and $2 \mathrm{~h}$ of incubation (Figs. 1 and 2). Whereas no or a low fluorescence signal was detected when Ag85B and
HspX were used alone without OCN, interestingly, most cells showed a single-antigen staining pattern with little
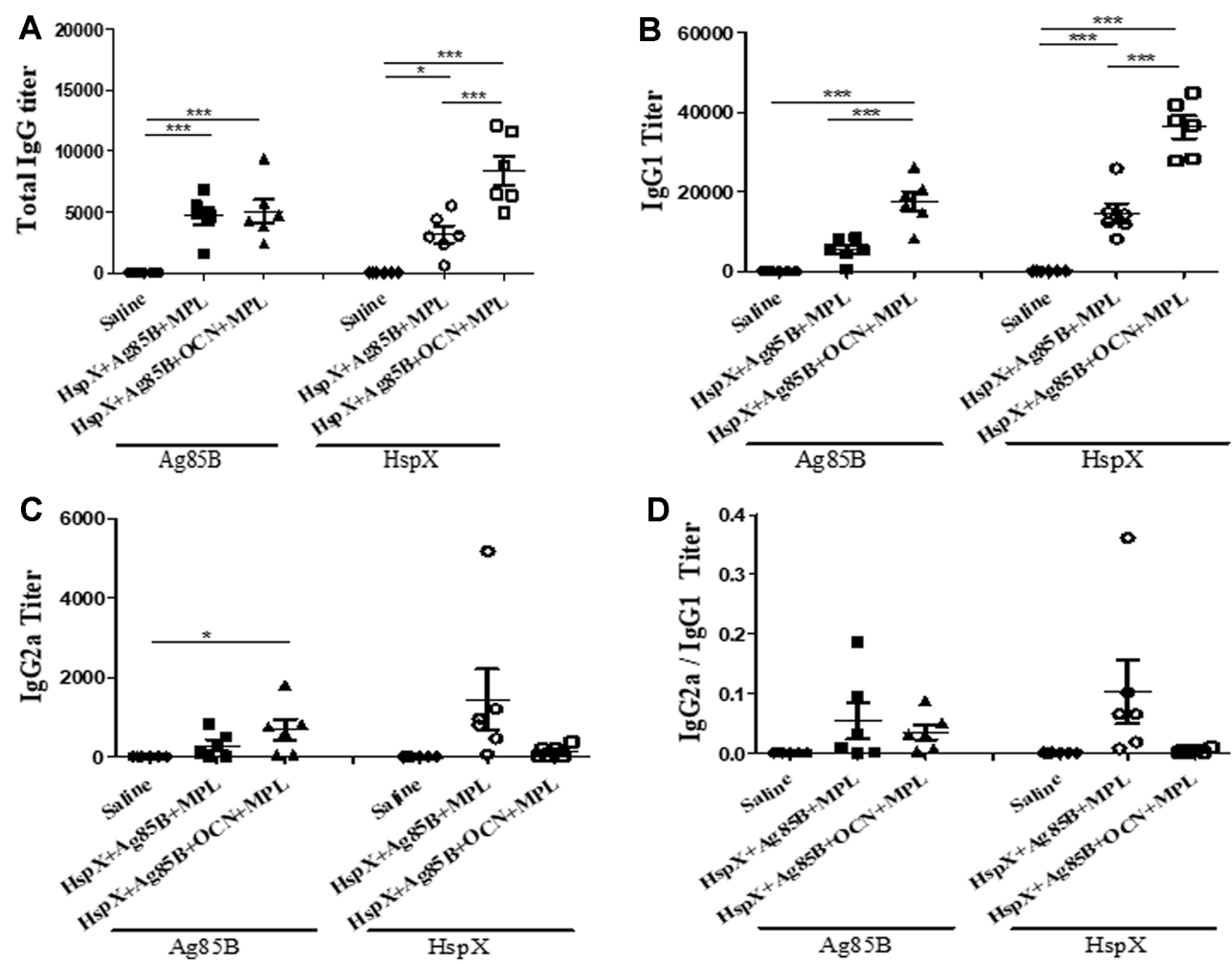

Fig. 3. OCNs influence antibody titer when combined with HspX, Ag85B and MPL.

(A-C) For serological responses to Ag85B or HspX, sera were collected for measuring specific total IgG titer, IgG1 and IgG2a. (D) The ratio of IgG2a to IgG1 is also shown. The significance of differences between groups was determined by one-way analysis of variance (ANOVA). ${ }^{*} p<0.05$, ${ }^{* *} p<0.01$ and ${ }^{* * *} p<0.001$. 
overlapping of both antigens, suggesting that each antigen was separately taken up by each individual cell. Therefore, OCNs effectively promoted protein antigen uptake by professional APCs.

\section{Immunogenicity of OCNs with HspX, Ag85B and MPL}

To study the influence of OCNs on the outcome of immune response, mice were immunized and serum and splenocytes were collected as described in Materials and Methods. For serological response, specific antibody against Ag85B and HspX in serum were measured. Because IgG2a and IgG1 titer corresponds to Th2 and Th1 immune responses, the titer of these two isotypes were compared. Overall, adding OCNs to the vaccine formulas induced higher specific IgG and IgG1 titer than antigens and MPL alone and this is more evident in HspX specific antibody titer (Figs. 3A and 3B). More importantly, the results showed that IgG1 titer in mice receiving $\mathrm{HspX}+\mathrm{Ag} 85 \mathrm{~B}+\mathrm{OCN}+\mathrm{MPL}$ was higher than the other 2 groups that received only PBS or $\mathrm{HspX}+\mathrm{Ag} 85 \mathrm{~B}+\mathrm{MPL}$, while there was no significant difference in the IgG2a titer between the $\mathrm{HspX}+\mathrm{Ag} 85 \mathrm{~B}+\mathrm{OCN}$ $+\mathrm{MPL}$ and HspX+Ag85B+MPL groups (Fig. 3C). This is true for both antigens. Additionally, the ratio of IgG2a to IgG1 also demonstrated that there are no statistical significant differences between all groups in both Ag85B and HspX stimulation (Fig. 3D).

For the cytokine profiles, splenocytes from immunized mice were restimulated with HspX and Ag85B separately in vitro. High IL-5 production is associated with Th2mediated immune response, while IFN- $\gamma$ production is representative of Th1-mediated immune response. Under restimulation with Ag85B, IL-5 production in the HspX+ $\mathrm{Ag} 85 \mathrm{~B}+\mathrm{OCN}+\mathrm{MPL}$ group was significantly lower than in the HspX+Ag85B+MPL group, but the difference between them was not observed in HspX restimulation (Fig. 4A). For IFN- $\gamma$ production, the group receiving $\mathrm{HspX}+\mathrm{Ag} 85 \mathrm{~B}+$ OCN+MPL showed a significantly higher level of IFN- $\gamma$ than that receiving $\mathrm{HspX}+\mathrm{Ag} 85 \mathrm{~B}+\mathrm{MPL}$ in both Ag85B and HspX restimulation (Fig. 4B). The results of IL-2 production in both $\mathrm{Ag} 85 \mathrm{~B}$ and $\mathrm{HspX}$ restimulation also followed the same trend with IFN- $\gamma$ production (Fig. 4C). Taken together, OCNs in combination with MPL enhanced Th1 cytokine production while decreasing Th2 cytokine production in splenocyte restimulation assay.

\section{OCN Enhanced Cytotoxic CD8 ${ }^{+} \mathrm{T}$ Cell Activation to Mtb Protein}

To study the effect of OCNs on cytotoxic $\mathrm{CD}^{+} \mathrm{T}$ cell activation, splenocytes were restimulated with Ag85B or
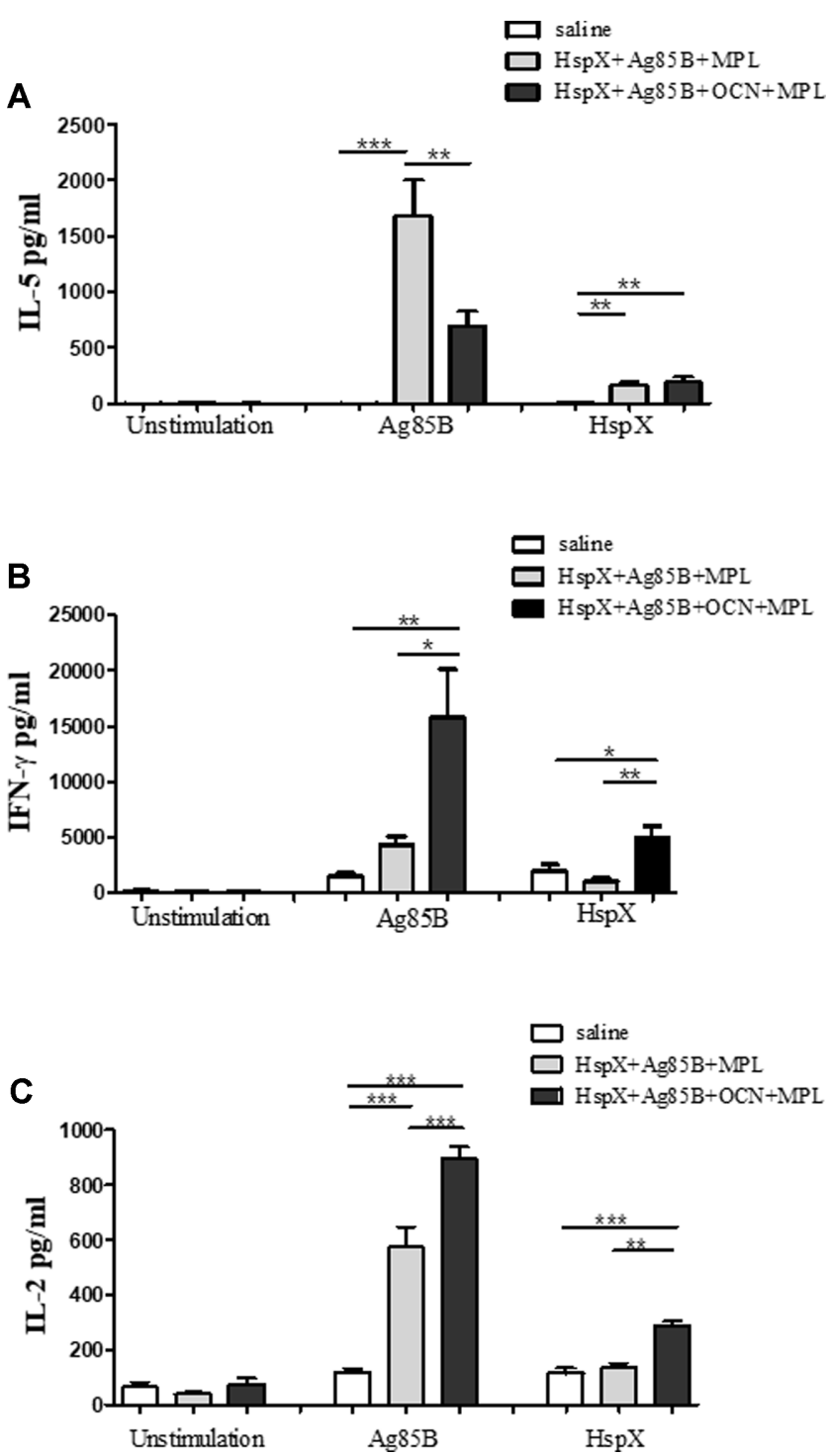

Fig. 4. OCNs increase IFNg but reduce IL-4 in splenocyte restimulation assay.

Splenocytes were stimulated with Ag85B $(10 \mu \mathrm{g} / \mathrm{ml})$ or HspX $(10 \mu \mathrm{g} / \mathrm{ml})$ separately. (A-C) Culture supernatant was collected and used to measure IL- 5 and IFN- $\gamma$ at $72 \mathrm{~h}$, and IL- 2 at $48 \mathrm{~h}$ by ELISA. Data obtained from each group represent the means \pm SEM. The significance of differences between groups was determined by one-way analysis of variance (ANOVA). ${ }^{*} p<0.05,{ }^{* *} p<0.01$ and ${ }^{* * *} p<0.001$.

HspX and stained for CD3, CD8 and granzyme B (Figs. 5A and $5 B)$. In HspX restimulated condition, the percentages of $\mathrm{CD}^{+} \mathrm{T}$ cells that produced granzyme B in HspX+Ag85B $+\mathrm{OCN}+\mathrm{MPL}$ immunized mice were significantly higher than the other groups. With the restimulation of Ag85B, the percentage of $\mathrm{CD}^{+}$granzyme $\mathrm{B}^{+}$cells in the groups of 

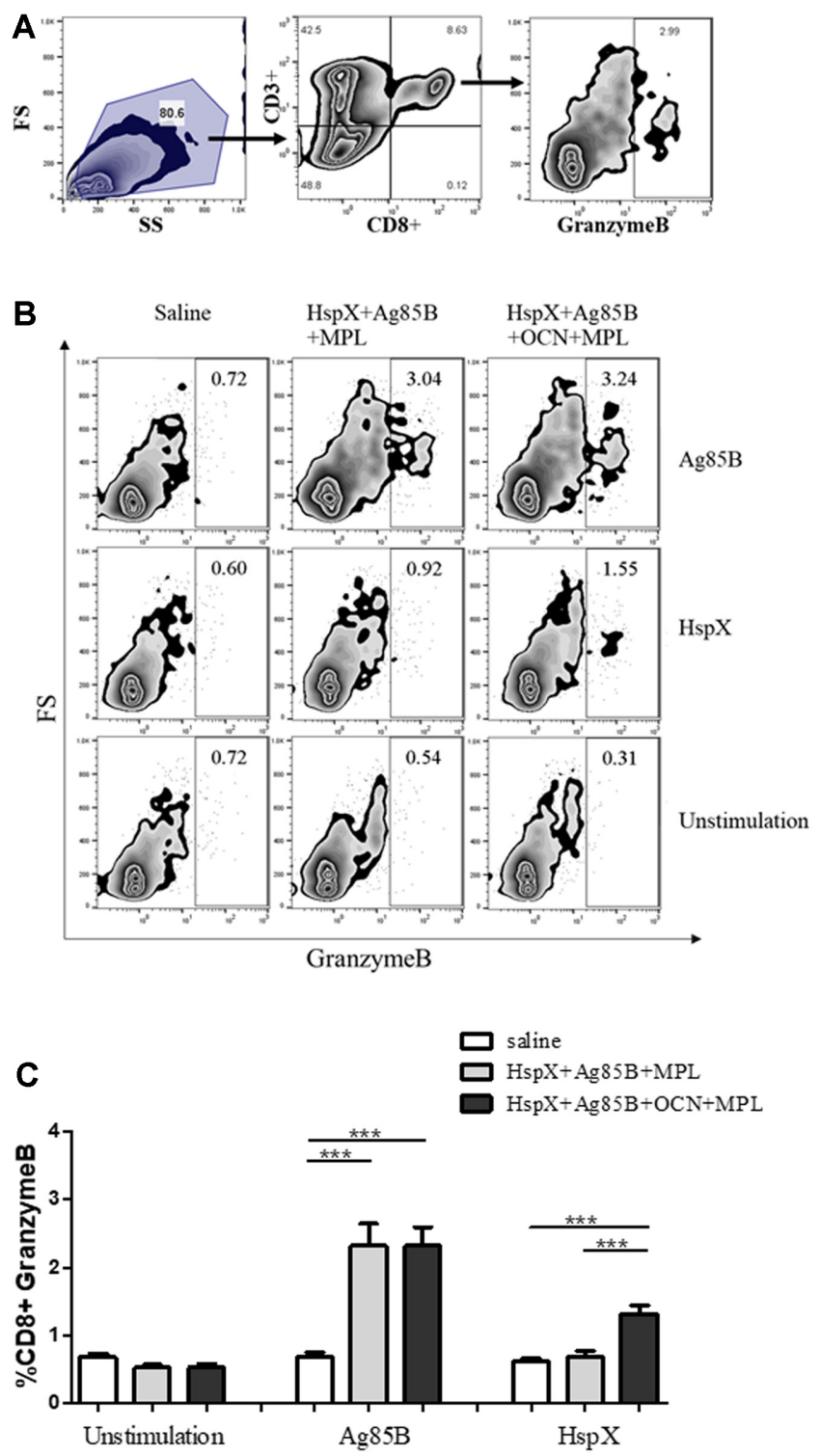

Fig. 5. OCNs augment the frequencies of activated cytotoxic CD8+ T cells.

$(\mathbf{A}-\mathbf{B}) \mathrm{BALB} / \mathrm{c}(n=6)$ mice were immunized as indicated above. One week after the last immunization, splenocytes were harvested and stimulated with Ag85B (10 $\mu \mathrm{g} / \mathrm{ml})$ and HspX $(10 \mu \mathrm{g} / \mathrm{ml})$ separately in vitro for $72 \mathrm{~h}$ in the presence of brefeldin A for the last $4 \mathrm{~h}$. Cells were collected for intracellular granzyme B staining. Representative flow cytometry results are shown. (C) The percentage of Ag85B and HspX specific $C D 8^{+} \mathrm{T}$ cells producing granzyme B were summarized. Data represent the means \pm SEM. The significance of differences between groups was determined by one-way analysis of variance (ANOVA). ${ }^{* * *} p<0.001$.

$\mathrm{HspX}+\mathrm{Ag} 85 \mathrm{~B}+\mathrm{OCN}+\mathrm{MPL}$ and $\mathrm{HspX}+\mathrm{Ag} 85 \mathrm{~B}+\mathrm{MPL}$ were similar and higher than the control saline group (Fig. 5C).
This result indicated that OCNs augmented the frequencies of granzyme B-producing cytotoxic CD8 ${ }^{+} \mathrm{T}$ cells to HspX.

\section{OCNs Combined with HspX, Ag85B and MPL were Not Sufficient to Protect Mice against Mtb Challenge}

To evaluate the protective efficacy induced by subunit TB proteins combined with OCNs and MPL with or without priming with BCG, immunized mice were respiratory challenged with virulent Mtb H37Rv strain six weeks after the last immunization. Changes in body weight were measured during the experimental time line and there were no significant differences in the body weight among all groups and both immunization strategies showed similar results (Figs.6A and 7A). Six weeks after the challenge, the bacterial burden in the lung and spleen was evaluated. In the two experiments, BCG was used as a positive control standard. The numbers of bacteria in the lung and spleen of BCG-immunized mice were significantly lower when compared with the PBS control group. Unfortunately, the bacterial CFUs in both organs of other tested groups were not decreased when compared with the PBS group (Figs. 6B and 6C). In the BCG priming regimen, adding $\mathrm{Ag} 85 \mathrm{~B}+\mathrm{HspX}+\mathrm{OCN}+\mathrm{MPL}$ did not reduce the bacterial burden further than those in the BCG alone (Figs. 7B and 7C). These results indicated that the prototype TB vaccine combining OCNs with two recombinant proteins was not sufficient to induce protective immunity against $\mathrm{Mtb}$ infection in either primary vaccination or prime-boost strategies.

\section{Discussion}

In this study, we evaluated a novel type of nanoparticle (oxidized carbon nanoparticles or OCNs) as a delivery system for subunit TB vaccine. Because cell-mediated immune responses and Th1 responses are considered the major immune response against $\mathrm{Mtb}$ infection, and designing a better delivery system that targets and enhances this type of immune response is desirable for a new TB vaccine candidate $[4,9,24]$.

APCs are cells that process and present antigens via MHC class I and II to T cells. Targeting antigen to these cells and engineering the route of antigen processing can drive appropriate immune responses. Consistent with the previous reports, OCNs were efficiently taken up by macrophages. Previous reports proposed that OCNs can generate transient pores on the lipid membrane of endosomes where they then leak into the cytosol and become localized [18]. Using this rationale, we expected 
A
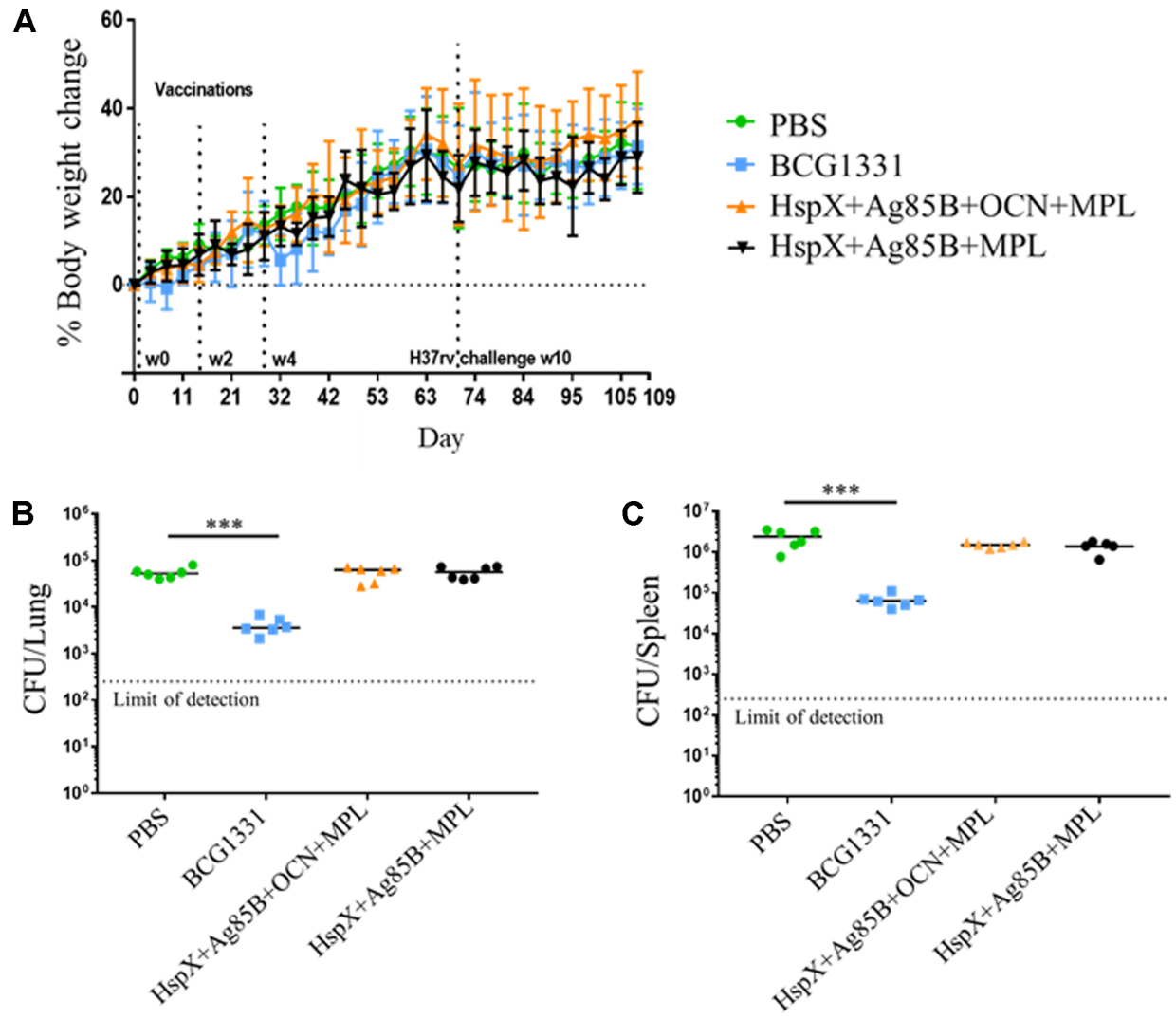

Fig. 6. Protective efficacy of OCN in combination with HspX, Ag85B and MPL in prime-boost strategy.

CB6F1/Crl female mice were immunized with PBS, HspX+Ag85B+MPL and HspX+Ag85B+OCN+MPL subcutaneously for 3 times at 2 weeks interval, except for BCG1331 $\left(5 \times 10^{6} \mathrm{CFU} /\right.$ mouse $)$ that was immunized as a single dose at the last immunized time. Six weeks later, mice were aerosol challenged with $\mathrm{H} 37 \mathrm{Rv} \mathrm{Mtb}(100 \mathrm{CFU} /$ mouse). (A) The changes in body weight were monitored. Each line corresponds to a single experimental group. (B-C) The protective efficacy was determined and demonstrated as the number of Mycobacterium CFU per lung and spleen at 6 weeks after challenge. Data are presented as mean \pm SD. The significance of differences between groups was determined by one-way analysis of variance (ANOVA). ${ }^{* * *} p<0.001$.

that the protein antigens delivered by OCNs would be processed and presented to $\mathrm{CD} 8^{+} \mathrm{T}$ cells as well as $\mathrm{CD} 4^{+} \mathrm{T}$ cells. Consistent with this hypothesis, the frequency of granzyme B-producing $\mathrm{CD}^{+} \mathrm{T}$ cells is higher when OCNs were combined with recombinant antigens, indicating that OCNs possibly drive antigen presentation to $\mathrm{CD} 8^{+} \mathrm{T}$ cells. This mechanism was also reported when PLGA was used as a delivery system [14].

Currently, one of the most advanced TB vaccine candidates is based on BCG engineered to express Listeria monocytogenes listeriolysin $\mathrm{O}$ in the urease $\mathrm{C}$ deletion background (VPM1002). This VPM1002 candidate vaccine facilitates endosomal escape of BCG vaccine, resulting in leakage of bacterial DNA into cytosol which triggers activation of inflammasome, and increased autophagy and apoptosis [25-27]. The proposed protective mechanisms of this vaccine are the effective $\mathrm{CD}^{+} \mathrm{T}$ cell priming through antigen cross-presentation by DC and well-balanced Th1 and Th17 responses [25, 26, 28, 29]. Therefore, it is highly possible to target balanced Th1/Th17 responses together with $\mathrm{CD} 8^{+} \mathrm{T}$ cell response and acquired protective immunity against $\mathrm{Mtb}$.

The reason that we used MPL in our vaccine system is to increase Th1 immune response. MPL, a Toll-like receptor (TLR4) agonist is one of the few licensed adjuvants for human patients. It is an attenuated version of lipopolysaccharide (LPS) with less toxicity that also maintains its immunostimulatory activity [30]. Signaling via TLR4 involves myeloid differentiation primary response 88 (MYD88)dependent and TIR-domain-containing adapter-inducing interferon- $\beta$ (TRIF)-dependent pathways, leading to robust proin-flammatory cytokines and type I interferon 

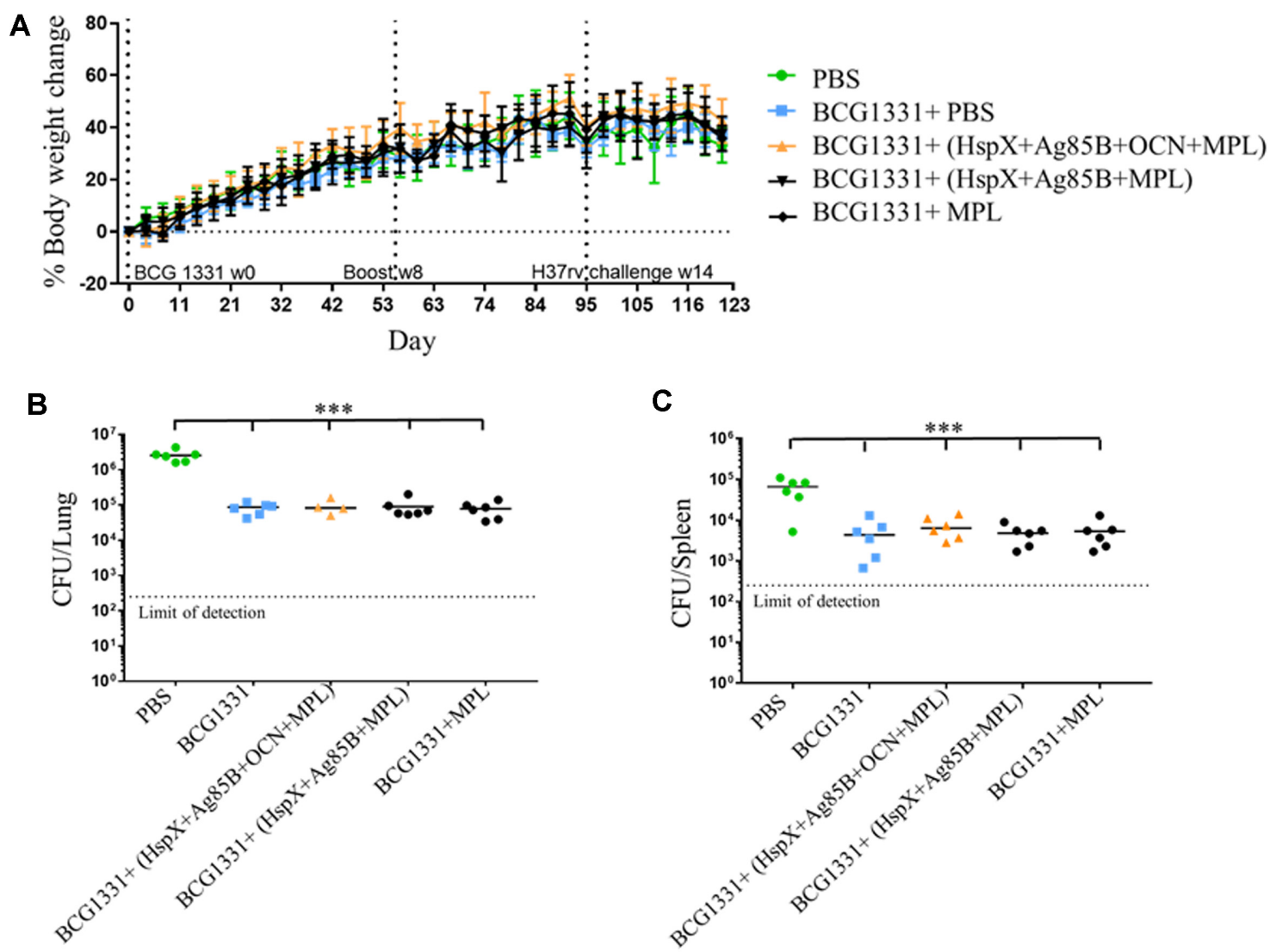

Fig. 7. Protective efficacy of OCNs in combination with HspX, Ag85B and MPL as a booster following BCG priming.

CB6F1/Crl female mice were primed with BCG1331 $\left(5 \times 10^{6} \mathrm{CFU} /\right.$ mouse $)$ on week 0 plus single dose of boost with corresponding vaccine candidates on week 8 . The control group received only one dose of PBS on week 0 . Six weeks later, mice were aerosol challenged with H37Rv Mtb (100 CFU/mouse). (A) The changes in body weight were monitored. (B-C) The protective efficacy was determined and demonstrated as the number of Mycobacterium CFU per lung and spleen at 6 weeks after challenge. Data are presented as mean $\pm \mathrm{SD}$. Each line corresponds to a single experimental group. The significance of differences between groups was determined by one-way analysis of variance (ANOVA). ${ }^{* *} p<0.001$.

production, respectively $[30,31]$. The bias of MPL toward TRIF signaling leads to low toxicity because of decreasing proinflammatory cytokines. MPL generally drives the immune responses toward a Th1 type [30]. Our cytokine profiles from specific antigen re stimulated splenocytes indicated that MPL induces Th1 immune response.

The subcutaneous layer lines the underside of the dermal layer, the layer consisting of high numbers of immune cells including lymphocytes, macrophages, and dermal DCs [32]. Therefore, vaccine administration through this route is expected to be captured by local APCs which drain to local lymph nodes for antigen presentation. Especially, DCs expressing langerin are dermal DCs that are well known to generate cross-presentation suitable for stimulating $\mathrm{CD} 8^{+} \mathrm{T}$ cells [33]. In addition, there are reports on TB vaccine using biopolymer poly (lactic-co-glycolic acid) (PLGA)-based vaccines that are injected via the subcutaneous route. This vaccine elicited Th1 immune response and protected mice from Mycobacterium infection [34, 35]. Therefore, the subcutaneous route was chosen for the subunit vaccine immunization.

Surprisingly, when OCNs were added to the vaccine formula, the level of Th1 signature cytokine, IFN- $\gamma$, was increased, while the level of Th2 cytokine, IL-5, was significantly reduced. This result suggested that OCNs can drive the immune response towards Th1. However, the cytokine profiles detected here did not correlate with specific antibody titer. The results of antibody titers from serum indicated that the response was polarized towards humoral immune response. The discrepancies between antibody isotypes and cytokine profiles are currently unknown. Recently characterized follicular helper T cell 
(Tfh) plays a more dominant role in humoral responses, especially in maintaining germinal center that is crucial for $B$ cell survival, isotype switching and antibody maturation via CD40L, IL-21, and IL-4 [36]. In our study, we did not characterize this subset of $\mathrm{T}$ cells or measure the Tfh key cytokines. Therefore, it is possible that OCNs may also affect local Tfh response, resulting in higher IgG1 titer than IgG2a. This possibility remains unanswered and needs further investigation.

Despite the novel vaccine formulation showing good immunogenicity, it was not correlated with an enhanced in vivo protection. The vaccine could not reduce the bacterial burden in the lung and spleen of Mtb challenged mice in both the priming and boosting strategies. One of the reasons we did not observe a protective response may lie in the recombinant antigens used in this study. It has been observed that fusing two different antigens when formulating the vaccine can result in enhanced protection compared to formulating them separately. For instance, it was shown that Ag85B-ESAT6 fusion protein and DDA/ MPL or DDA/TDB as adjuvants can induce protection against the Mtb challenge in guinea pigs and mice [37, 38]. Non-fusion proteins Ag85B and ESAT6 individually or in combination, on the other hand, did not confer protection [37]. The multi-stage subunit vaccine that consists of ESAT6-Ag85B-MPT64(190-198)-Mtb8.4-HspX combined with DDA/PolyI:C showed strong immunogenicity and longlasting protective efficacy against Mtb [39]. H56, a clinical trial Mtb protein consisting of Ag85A, ESAT-6, and Rv2660c (dormant state protein), conferred highly protective efficacy in both before and after exposure to Mtb [40].

In summary, the present study reveals that OCNs are a good subunit protein carrier for delivering protein into APCs such as macrophages. Moreover, OCNs mixed with two state proteins of Mtb, Ag85B and HspX, and MPL as adjuvant, increased cytotoxic $\mathrm{CD} 8^{+} \mathrm{T}$ cell activation. The responses were polarized to Th1 immune response characterized by cytokine profiles. Unfortunately, the immune responses elicited by this novel vaccine formulation did not confer protection in vivo against a challenge with Mtb.

\section{Acknowledgments}

This work was supported by the Thai Government Annual Budget and the Newton Fund-Institutional Links (UK), the National Nanotechnology Center (NANOTEC), NSTDA, Ministry of Science and Technology, Thailand, through its program of Research Network NANOTEC
(RNN) and PS is supported by the $100^{\text {th }}$ Anniversary Chulalongkorn University Fund for Doctoral Scholarship. The authors wish to acknowledge Dr. Patcharee Ritprajak for her technical help on dendritic cells.

\section{Conflict of Interest}

The authors have no financial conflicts of interest to declare.

\section{References}

1. Moreno-Mendieta SA, Rocha-Zavaleta L, Rodriguez-Sanoja R. 2010. Adjuvants in tuberculosis vaccine development. FEMS Immmunol. Med. Microbiol. 58: 75-84.

2. Andersen P, Doherty M. 2005. The success and failure of BCG-implications for a novel tuberculosis vaccine. Nat. Rev. Microbiol. 3: 656-662.

3. Nunes-Alves C, Booty MG, Carpenter SM, Jayaraman P, Rothchild AC, Behar SM. 2014. In search of a new paradigm for protective immunity to TB. Nat. Rev. Microbiol. 12: 289-299.

4. Ottenhoff TH, Kaufmann SH. 2012. Vaccines against tuberculosis: where are we and where do we need to go? PLoS Path. 8: e1002607.

5. Fehres CM, Unger WWJ, Garcia-Vallejo JJ, van Kooyk Y. 2014. Understanding the biology of antigen cross-presentation for the design of vaccines against cancer. Front. Immunol. 5: 149.

6. Kasturi SP, Pulendran B. 2008. Cross-presentation: avoiding trafficking chaos? Nat. Immunol. 9: 461-463.

7. Joshi VB, Geary SM, Salem AK. 2013. Biodegradable particles as vaccine delivery systems: size matters. AAPS J. 15: 85-94.

8. Smith DM, Simon JK, Baker JR, Jr. 2013. Applications of nanotechnology for immunology. Nat. Rev. Immunol. 13: 592-605.

9. Gregory AE, Titball R, Williamson D. 2013. Vaccine delivery using nanoparticles. Front. Cell. Infect. Microbiol. 3: 13.

10. Couvreur PV, C. 2006. Nanotechnology: Intelligent design to treat complex disease. Pharm. Res. 23: 1417-1450.

11. Demento SL, Cui W, Criscione JM, Stern E, Tulipan J, Kaech SM, et al. 2012. Role of sustained antigen release from nanoparticle vaccines in shaping the $\mathrm{T}$ cell memory phenotype. Biomaterials 33: 4957-4964.

12. van Dissel JT, Arend SM, Prins C, Bang P, Tingskov PN, Lingnau K, et al. 2010. Ag85B-ESAT-6 adjuvanted with IC31 promotes strong and long-lived Mycobacterium tuberculosis specific $\mathrm{T}$ cell responses in naïve human volunteers. Vaccine 28: 3571-3581.

13. Leleux J, Roy K. 2013. Micro and nanoparticle-based delivery systems for vaccine immunotherapy: an immunological and materials perspective. Adv. Healthc Mater. 2: 72-94.

14. Shen H, Ackerman AL, Cody V, Giodini A, Hinson ER, Cresswell P, et al. 2006. Enhanced and prolonged crosspresentation following endosomal escape of exogenous antigens 
encapsulated in biodegradable nanoparticles. Immunology 117: 78-88.

15. Hirosue S, Kourtis IC, van der Vlies AJ, Hubbell JA, Swartz MA. 2010. Antigen delivery to dendritic cells by poly(propylene sulfide) nanoparticles with disulfide conjugated peptides: Cross-presentation and T cell activation. Vaccine 28: 78977906.

16. Khademi F, Derakhshan M, Yousefi-Avarvand A, Tafaghodi M. 2018. Potential of polymeric particles as future vaccine delivery systems/adjuvants for parenteral and non-parenteral immunization against tuberculosis: A systematic review. Iran J. Basic Med. Sci. 21: 116-123.

17. Arayachukeat S, Palaga T, Wanichwecharungruang SP. 2012. Clusters of carbon nanospheres derived from graphene oxide. ACS Appl. Mat. Inter. 4: 6808-6815.

18. Arayachukiat S, Seemork J, Pan-In P, Amornwachirabodee K, Sangphech N, Sansureerungsikul T, et al. 2015. Bringing macromolecules into cells and evading endosomes by oxidized carbon nanoparticles. Nano Lett. 15: 3370-3376.

19. Mata-Haro V, Cekic C, Martin M, Chilton PM, Casella CR, Mitchell TC. 2007. The vaccine adjuvant monophosphoryl lipid a as a TRIF-biased agonist of TLR4. Science 316: 1628-1632.

20. Yuan Y, Crane DD, Simpson RM, Zhu Y, Hickey MJ, Sherman DR, et al. 1998. The 16-kDa $\alpha$-crystallin (Acr) protein of Mycobacterium tuberculosis is required for growth in macrophages. Proc. Natl. Acad. Sci. USA 95: 9578-9583.

21. Belisle JT, Vissa VD, Sievert T, Takayama K, Brennan PJ, Besra GS. 1997. Role of the major antigen of Mycobacterium tuberculosis in cell wall biogenesis. Science 276: 1420-1422.

22. Boonyatecha N, Sangphech N, Wongchana W, Kueanjinda P, Palaga T. 2012. Involvement of notch signaling pathway in regulating IL-12 expression via c-Rel in activated macrophages. Mol. Immunol. 51: 255-262.

23. Nguyen TNY, Padungros P, Wongsrisupphakul P, Sa-ArdIam N, Mahanonda R, Matangkasombut O, et al. 2018. Cell wall mannan of Candida krusei mediates dendritic cell apoptosis and orchestrates Th17 polarization via TLR-2/ MyD88-dependent pathway. Sci. Rep. 8: 17123.

24. Fletcher HA, Schrager L. 2016. TB vaccine development and the End TB Strategy: importance and current status. Trans. R. Soc. Trop. Med. Hyg. 110: 212-218.

25. Grode L, Seiler P, Baumann S, Hess J, Brinkmann V, Eddine AN, et al. 2005. Increased vaccine efficacy against tuberculosis of recombinant Mycobacterium bovis bacille Calmette-Guérin mutants that secrete listeriolysin. J. Clin. Invest. 115: 2472-2479.

26. Hoft DF, Blazevic A, Abate G, Hanekom WA, Kaplan G, Soler JH, et al. 2008. A new recombinant BCG vaccine safely induces significantly enhanced TB-specific immunity in human volunteers. J. Infect. Dis. 198: 1491-1501.

27. Nieuwenhuizen NE, Kaufmann SHE. 2018. Next-generation vaccines based on Bacille Calmette-Guerin. Front Immunol. 9: 121.
28. Winau F, Weber S, Sad S, de Diego J, Hoops SL, Breiden B, et al. 2006. Apoptotic vesicles crossprime CD8 T cells and protect against tuberculosis. Immunity 24: 105-117.

29. Desel C, Dorhoi A, Bandermann S, Grode L, Eisele B, Kaufmann SH. 2011. Recombinant BCG Delta ureC hly+ induces superior protection over parental BCG by stimulating a balanced combination of type 1 and type 17 cytokine responses. J. Infect. Dis. 204: 1573-1584.

30. Casella CR, Mitchell TC. 2008. Putting endotoxin to work for us: monophosphoryl lipid $\mathrm{A}$ as a safe and effective vaccine adjuvant. Cell Mol. Life Sci. 65: 3231-3240.

31. Awasthi S. 2014. Toll-Like receptor-4 modulation for cancer immunotherapy. Front Immunol. 5: 328.

32. Neeland MR, Shi W, Collignon C, Taubenheim N, Meeusen ENT, Didierlaurent AM, et al. 2016. The Lymphatic immune response induced by the adjuvant AS01: a comparison of intramuscular and subcutaneous immunization routes. $J$. Immunol. 197: 2704.

33. Leleux J, Roy K. 2013. Micro and nanoparticle-based delivery systems for vaccine immunotherapy: an immunological and materials perspective. Adv. Healthc. Mater. 2: 72-94.

34. Carlétti D, Morais da Fonseca D, Gembre AF, Masson AP, Weijenborg Campos L, Leite LCC, et al. 2013. A single dose of a DNA vaccine encoding apa coencapsulated with 6,6'trehalose dimycolate in microspheres confers long-term protection against tuberculosis in Mycobacterium bovis BCG-primed mice. Clin. Vaccine immunol. 20: 1162-1169.

35. Ha S-J, Park S-H, Kim H-J, Kim S-C, Kang H-J, Lee E-G, et al. 2006. Enhanced immunogenicity and protective efficacy with the use of interleukin-12-encapsulated microspheres plus AS01B in tuberculosis subunit vaccination. Infect. Immun. 74: 4954-4959.

36. Crotty S. 2014. T follicular helper cell differentiation, function, and roles in disease. Immunity 41: 529-542.

37. Olsen AW, Williams A, Okkels LM, Hatch G, Andersen P. 2004. Protective effect of a tuberculosis subunit vaccine based on a fusion of Antigen 85B and ESAT-6 in the aerosol guinea pig model. Infect. Immun. 72: 6148-6150.

38. Doherty TM, Olsen AW, Weischenfeldt J, Huygen K, D'Souza S, Kondratieva TK, et al. 2004. Comparative analysis of different vaccine constructs expressing defined antigens from Mycobacterium tuberculosis. J. Infect. Dis. 190: 2146-2153.

39. Niu H, Peng J, Bai C, Liu X, Hu L, Luo Y, et al. 2015. Multistage tuberculosis subunit vaccine candidate LT69 provides high protection against Mycobacterium tuberculosis infection in mice. PLoS One 10: e0130641.

40. Aagaard C, Hoang T, Dietrich J, Cardona P-J, Izzo A, Dolganov $G$, et al. 2011. A multistage tuberculosis vaccine that confers efficient protection before and after exposure. Nat. Med. 17: 189-194. 